\title{
Convergence Analysis of Spectrum Balancing Algorithms for DSL Networks
}

\author{
Marcio Monteiro, Cleyton Muto, Francisco Müller and Aldebaro Klautau
}

\begin{abstract}
Resumo-Nos últimos anos, muito esforço foi alocado em pesquisa e desenvolvimento de técnicas de gerenciamento dinâmico de espectro (Dynamic Spectrum Management - DSM) com o objetivo de aumentar o alcance e a taxa de transmissão de dados de sistemas de linha digital de assinante (Digital Subscriber Line - DSL). O gerenciamento dinâmico de espectro reduz o efeito da interferência eletromagnética (crosstalk) induzida nas linhas DSL através do uso de informacões sobre o estado atual da rede. A maioria dos algoritmos já desenvolvidos são iterativos. Se cada iteração necessitar que os modems DSL envolvidos se reconfigurem, incluindo suas densidades espectrais de potências, o serviço será interrompido durante esse período de reconfiguração. Por essa razão, a aplicação prática das técnicas de DSM é altamente dependente da taxa de convergência. Neste artigo, um estudo sobre a convergência de algoritmos DSM foi realizado considerando diferentes cenários, de forma a avaliar o comportamento do sistema para diferentes situações. Foi descoberto em nossos experimentos que, sem cuidado especial, alguns algoritmos podem levar até $\mathbf{2 0}$ minutos para convergir para o cenário de pior caso (com muita interferência de crosstalk), considerando as limitações dos padrões DSL atuais.
\end{abstract}

Palavras-Chave-Linha digital do assinante, gerênciamento dinâmico de espectro, balanceamento de espectro, convergência.

Abstract-During the last years, a lot of effort has been put into the research and development of dynamic spectrum management (DSM) techniques to increase the range and data rate of digital subscriber lines (DSL) systems. Dynamic spectrum management mitigates the effects of the crosstalk interference on DSL lines by using information about the current state of the DSL network. Most of the developed algorithms are iterative. If the iterations require the involved DSL modems to reconfigure themselves, including their power spectral density, the service will be interrupted during the reconfiguration. Therefore, the practical use of DSM techniques is highly dependent on its convergence rate. In this paper, a study of DSM algorithms convergence was performed considering different scenarios, in order to assess the system behavior for different situations. It was found in our experiments that, without special care, some algorithms can take up to 20 minutes to converge in the worst scenario (with very high crosstalk interference), considering the limitations of current DSL standards.

Keywords-Digital subscriber line (DSL), dynamic spectrum management (DSM), spectrum balancing, convergence.

\section{INTRODUCTION}

Digital subscriber lines (DSL) is a very cost-effective technology to deliver broadband access, as it uses the already

Marcio Monteiro, Cleyton Muto, Francisco Müller and Aldebaro Klautau are with the Laboratório de Processamento de Sinais, Instituto de Tecnologia, Universidade Federal do Pará, Belém, Brazil. E-mails: \{marciomm,cleyton,fmuller,aldebaro\}@ufpa.br. This work was partially supported by the Research and Development Center, Ericsson Telecommunications S.A., Brazil. existing copper infrastructure. For this reason, it plays an important role to deliver broadband access around the world. In 2008, only in Brazil, the ADSL (asymmetric DSL) technology represented $62 \%(7,086,750$ subscribers) of the market share among all broadband technologies [1].

However, the lines sharing the same cable bundle suffers from the mutual electromagnetic interference called crosstalk. Crosstalk is one of the main impairments for DSL systems [2] and it can severely impact their capacity. In order to address this issue, dynamic spectrum management (DSM) emerged as a promising approach for crosstalk mitigation or cancellation in DSL networks. Moreover, DSM methods have been investigated as an alternative solution to optimize spectral utilization by exploiting multi-user coordination techniques, consequently boosting data rate transmission while reducing crosstalk effects and possibly the power consumption [3]-[5].

As a result, many DSM algorithms appeared in the literature [6]-[10], each one with its own pros and cons. Naturally, comparisons between those algorithms became important for those interested in investigating or deploying DSM technology. Usually, the figures of merit used to compare them, like performance, complexity and network information (e.g. [11]), focus on the "spectrum management" part of DSM, that is, the main concern is to evaluate the possible achievable data rates by varying the transmitted power spectrum densities (PSD) of the modems. But one aspect that is often neglected is the fact that most DSM algorithms are iterative and each iteration may require the modems involved to spend some time reconfiguring themselves to follow the new transmission PSDs.

Therefore, specially when the iteration involves retraining the modem, the practical use of DSM techniques depends strongly on the number of iterations required for convergence and also on the time spent on each iteration. This work focuses on these "dynamic" aspects of DSM, which have not been carefully studied in the DSM literature, but that are important for practical deployments of DSM.

The remainder of this paper is organized as follows. Section II briefly introduces the DSL system model adopted and defines notation. Section III formalizes a metric for comparing the dynamic of DSM algorithms. Section IV shows numerical simulations and Section V presents the final conclusions.

\section{SySTEM MODEL}

The scenario for the use of DSM algorithms in this work is a copper access binder, which consists of $N$ users (i.e. lines) equipped with DSL transceivers. Each transceiver employs discrete multitone modulation (DMT) and operates 
over a twisted-pair line with $K$ independent parallel subchannels (tones) [12]. By assuming only frequency division duplex (FDD) DMT transmission, where upstream and downstream frequency bands are non-overlapping, only the farend crosstalk (FEXT) is assumed to be present. The near-end crosstalk (NEXT) influence is neglected during simulations. For a description of FEXT and NEXT, one can refer to [2], [12].

The problem of bit allocation per tone $k$ for user $n$ can be expressed as follows

$$
\begin{aligned}
b_{n}^{k} & =\log _{2}\left[1+\frac{S N R_{n}^{k}}{\Gamma}\right] \\
& =\log _{2}\left[1+\frac{s_{n}^{k}\left|h_{n, n}^{k}\right|^{2}}{\Gamma\left(\sigma_{n}^{k}+\sum_{m \neq n} s_{m}^{k}\left|h_{n, m}^{k}\right|^{2}\right)}\right]
\end{aligned}
$$

where

- $b_{n}^{k}$ is the achievable bitloading on tone $k$ for user $n$;

- $S N R_{n}^{k}$ is the signal-to-noise ratio on tone $k$ for user $n$;

- $\Gamma$ denotes the signal-to-noise ratio gap, which is a function of the desired bit error rate (BER);

- $s_{n}^{k}$ denotes the power allocated by user $n$ at tone $k$;

- $\sigma_{n}^{k}$ represents the background noise power on tone $k$ at receiver $n$;

- $\left|h_{n, n}^{k}\right|^{2}$ denotes the square-magnitude of the direct channel gain for user $n$ at tone $k$; and

- $\left|h_{n, m}^{k}\right|^{2}$ denotes the square-magnitude of the far-end crosstalk channel from transmitter $m$ to receiver $n$ at tone $k$.

The achievable bit rate of user $n$ can be expressed as

$$
R_{n}=f_{\mathrm{s}} \sum_{k=1}^{K} b_{n}^{k},
$$

where $f_{\mathrm{s}}$ represents the symbol rate of the DMT transceivers [13], which has a typical value of 4000 symbols/s.

\section{ITERATIVE Algorithms AND CONVERGENCE METRIC}

The computational cost to find the optimal PSD solution is intractable ${ }^{1}$ for a network with a large number of users. Hence, sub-optimal algorithms are preferred for optimizing large networks. This work analyzes two DSM algorithms classified (see [13]) as level 1 (Lv. 1) and three DSM Lv. 2 algorithms, totalizing five algorithms. It is assumed that all of them adopt the rate maximization mode of operation. These algorithms are:

- Iterative Water-Filling (IWF [6]) - Lv. 1

- Iterative Spectrum Balancing (ISB [7]) - Lv. 2

- Successive Convex Approximation for Water-Filling (SCAWF [9]) - Lv. 1

- Successive Convex Approximation for Low-complExity (SCALE [9]) - Lv. 2

- Semi-blind Spectrum Balancing (2SB [10]) - Lv. 2

${ }^{1}$ Considering that the only algorithm with an optimality proof [14], [15] is intractable.
All these algorithms are iterative and thus require many individual PSD mask recalculations and adaptations in order to reach their best performance. This number of PSD recalculations and adaptations is accounted here as the number of iterations. The iteration event itself is said to have occurred when every managed transceiver (modem) has updated its own PSD mask.

The PSD mask redefinition process can be triggered autonomously in a DSM Lv. 1 architecture (each transceiver has freedom/independence to decide the right moment to do it), or by a spectrum management center (SMC) agent in the centralized architecture typically assumed by DSM Lv. 2 techniques [4]. However, the dichotomic classification of algorithms in Lv. 1 and 2 has drawbacks. The SCALE algorithm, for example, was proposed as a distributed algorithm, which receives some information (the crosstalk gains) from the SMC. Hence, it is not a completely centralized or distributed algorithm. This issue will be discussed later on. This section is concerned only with evaluating the number of iterations each algorithm requires for convergence, not taking in account whether these iterations take place in the SMC or in the modems, in a distributed way.

Increasing the number of iterations for a given algorithm improves its performance in rate capacity until it converges. Fig. 1 shows an example of this behavior, where a greater number of iterations leads to a better (higher) set of achievable rates.

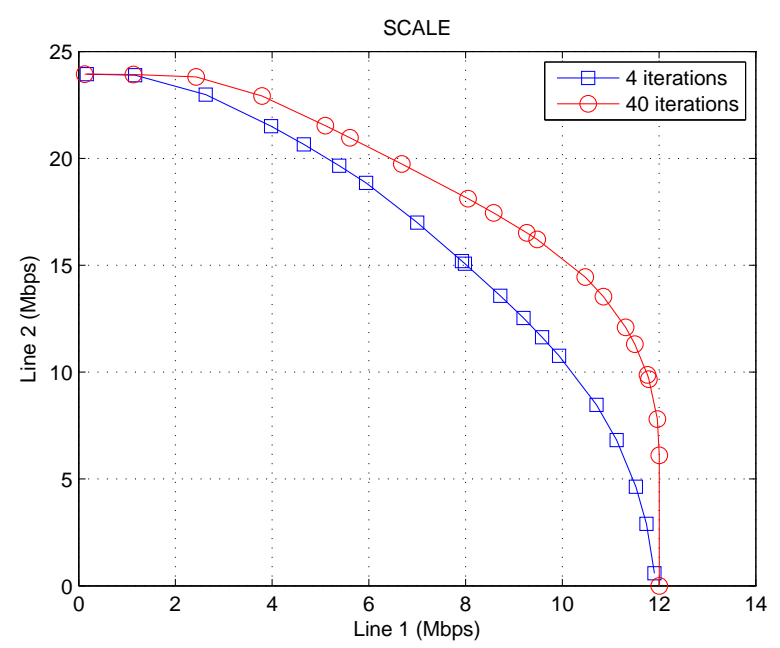

Fig. 1. The influence of the number of iterations over the rate region for the SCALE algorithm.

Hence, a natural question would be: how many iterations are necessary for a given algorithm to achieve its best performance (i.e. to converge)? As it is going to be shown in Section IV, the answer can vary widely for different algorithms, but it is important to keep it small when considering the deployment of DSM technology. If the iteration requires PSD reshaping through retraining the modem, the process in current transceivers takes some time to complete (around 20 to $40 \mathrm{sec}-$ onds in our experiments with different commercial modems). Meanwhile, the service is interrupted. As a result, an algorithm with slow convergence, even if it has low complexity, may take 
a longer time to reach the expected performance.

\section{A. The convergence criterion}

A straightforward convergence criterion would be the following: when two consecutive iterations result in exactly the same PSD masks, the algorithm is said to have converged. In particular for rate maximization algorithms (the use-case of this work), it is possible (and simpler) to keep track of the bit rate variations instead of the PSD masks convergence. Fig. 2 shows an example of typical rate evolution with the number of iterations for some 2-users scenario.

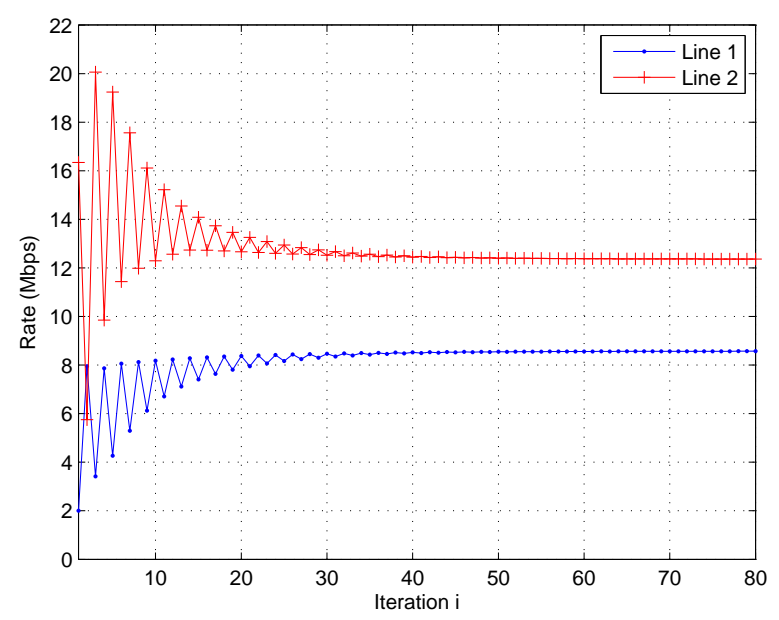

Fig. 2. Example of rate convergence for two lines (users) in the same binder.

From Fig. 2 it is possible to notice the rate variations get reduced as the number of iterations increases. We can say the absolute convergence is met when no variations occur anymore (i.e. two consecutive iterations result in the exactly same rates).

In practice, a convergence threshold $\Delta_{R}>0$ is assumed, as there is no convergence proof for the DSM algorithms considered. In this work we use $\Delta_{R}=f_{s}$, which means a variation equal to one bit in a QAM constellation of the DMT. Fig. 3 depicts a different view of Fig. 2, making explicit the rates variations (derivative) being reduced along the iterations until it gets lower than the adopted convergence limit $\left(\Delta_{R}=4 \mathrm{Kbps}\right)$.

In a more general (and formal) way, a DSM algorithm is said to have converged when two consecutive iterations make (3) a true statement:

$$
\left|\frac{d R_{n}(i)}{d i}\right| \leq \Delta_{R}, \quad \forall n
$$

where $i$ denotes the iteration counter.

\section{NumericAl RESUlts}

This section compares the convergence of different DSM algorithms. A near-far access network topology was used, as depicted in Fig. 4, which consists of $24 \mathrm{AWG}$ cables and transceivers located at the central office $(\mathrm{CO})$, at the remote terminal (RT) side and at the customer premises (CP) side.

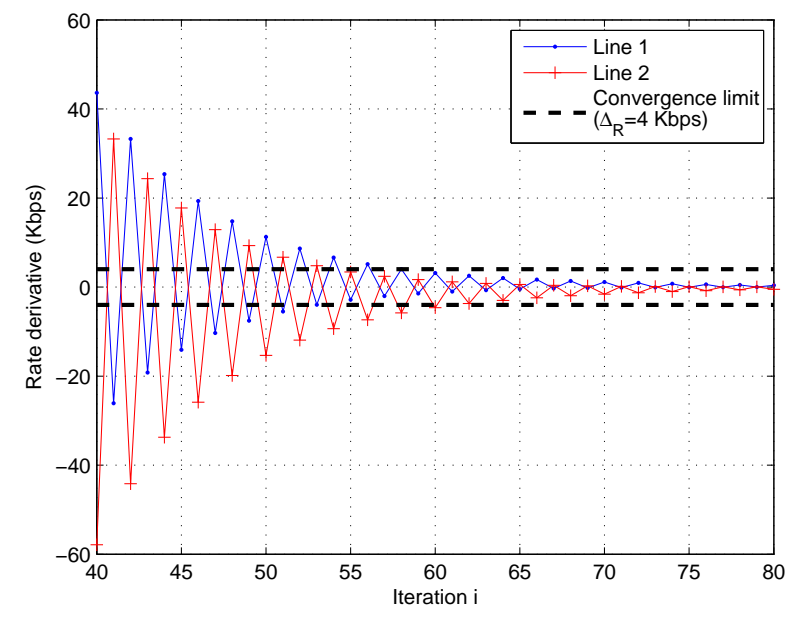

Fig. 3. Rate derivative corresponding to Fig. 2. It can be seen convergence in 62 iterations.

The downstream transmission is the object of interest. This topology was chosen because it is one of the most challenging scenarios for DSM in downstream transmission, as the RT lines generate high crosstalk to $\mathrm{CO}$ lines, eventually limiting their performance.

All considered DSL transceiver units correspond to ADSL2+ modems, a technology that is replacing ADSL for the home access. The simulation assumes a background noise consisting of AWGN with $-140 \mathrm{dBm} / \mathrm{Hz}$, SNR-gap of $9.75 \mathrm{~dB}$ for a BER $<10^{-7}$, coding gain of $3.5 \mathrm{~dB}$ and a total transmit power for each modem of $19.4 \mathrm{dBm}$.

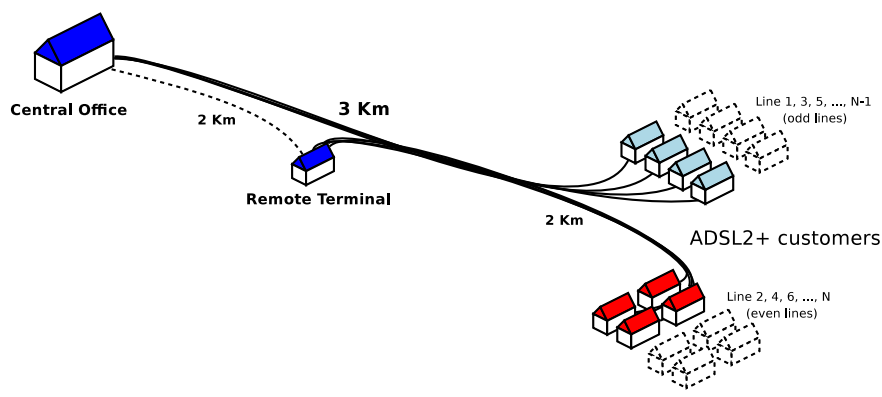

Fig. 4. The $N$-users DSL topology considered for simulations.

In order to assess the convergence behavior of these algorithms with respect to the number of users, simulations considering up to 40-users scenarios were performed. For the sake of the explanation, the odd lines (Line 1, 3, etc.) represent those connected to the central office, and the even ones (Line 2, 4 , etc.) to the remote terminal.

A convergence analysis of DSM Lv. 2 algorithms in a 4-users scenario is depicted in Fig. 5. According to the convergence criterion adopted in (3), ISB was able to converge in 7 iterations, SCALE in 61 and 2SB in 19. Besides, it is also possible to notice that the algorithms ISB and SCALE have reached an "equilibrium state", where no bit rate variations occurs anymore, as iterations goes by after a determined point. The 2SB algorithm, however, does not reach that equilibrium, 
presenting oscillations all over the iterations. Our simulations have shown that this behavior gets reduced when increasing the number of users, but it is always present. In spite of that, 2SB reaches convergence according to the adopted criterion.

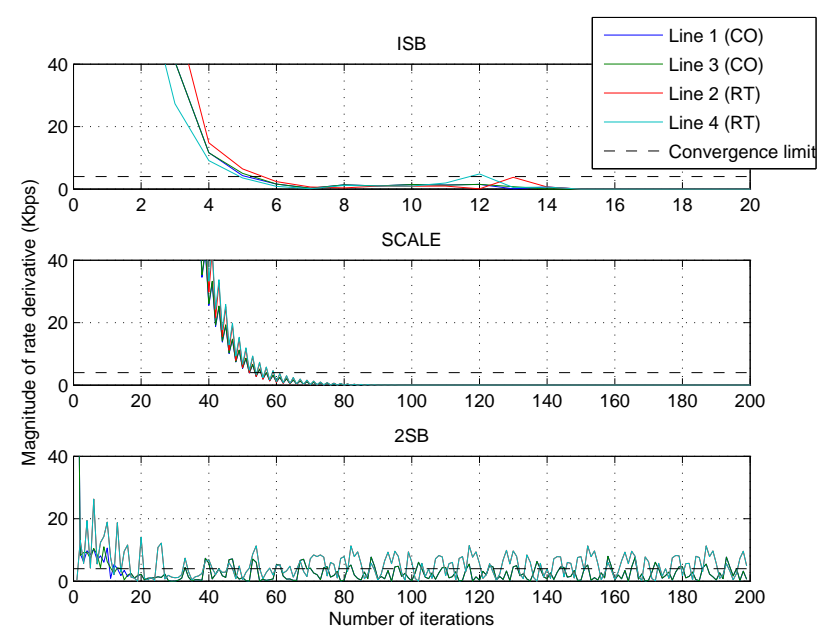

Fig. 5. 4-users convergence of DSM level 2. From top to bottom: ISB SCALE and 2SB.

The DSM Lv. 1 algorithms SCAWF and IWF had a rather quick convergence rate, up to 4 iterations. Their convergence rate is also constant with respect to the number of users, presenting minimum variations between 4 -users and 40 -users scenarios.

Table I shows in details the convergence rate of all algorithms considered in this work, for the different scenarios (4 to 40-users scenarios).

TABLE I

ITERATIONS TO CONVERGENCE, FOR $\Delta_{R}=4000$ BPS

\begin{tabular}{|c||c|c|c|c|c|}
\hline N. users & IWF & SCAWF & ISB & SCALE & 2SB \\
\hline \hline 4 & 3 & 4 & 7 & 61 & 23 \\
\hline 6 & 3 & 4 & 16 & 47 & 23 \\
\hline 8 & 3 & 4 & 23 & 43 & 22 \\
\hline 10 & 3 & 4 & 21 & 41 & 22 \\
\hline 20 & 3 & 4 & 20 & 35 & 25 \\
\hline 30 & 3 & 4 & 19 & 35 & 27 \\
\hline 40 & 3 & 3 & 18 & 35 & 27 \\
\hline
\end{tabular}

Fig. 6 gives a graphical view of Table I. For DSM Lv. 2 algorithms, it is possible to notice that the total number of iterations for convergence seems to stabilize after a certain point ( $N \geq 30$ ). For DSM Lv. 1 algorithms, they present almost constant convergence rate. Hence, from the convergence point of view, the DSM algorithms do not suffer from scalability problems, which is a very interesting property for DSL service providers with large networks.

Although SCALE has good capacity performance and low complexity, our simulations showed that it suffers from very slow convergence. In average, 40 PSD mask adaptations per user are required. In this case, instead of using SCALE in a distributed architecture, as proposed by its authors, the algorithm may be practical only in a centralized architecture, where

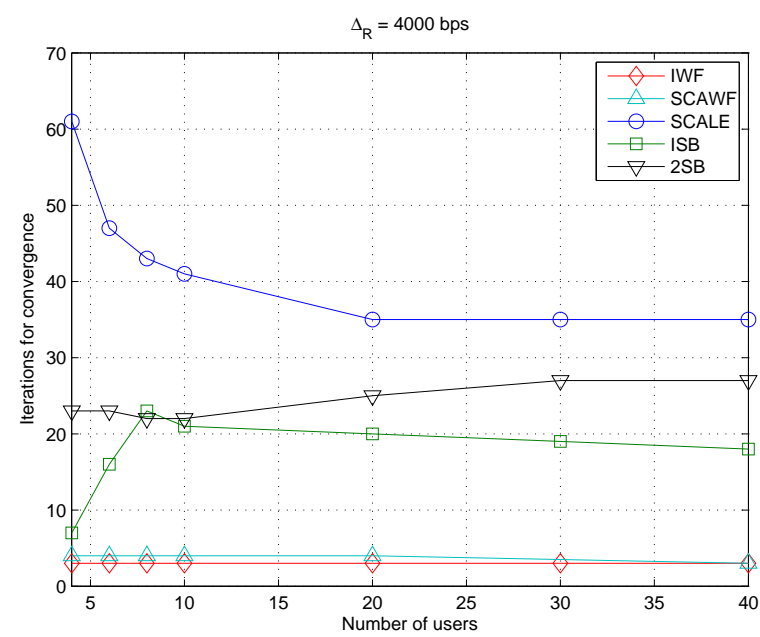

Fig. 6. Convergence comparison between different spectrum balancing algorithms assuming $\Delta_{R}=4 \mathrm{Kbps}$.

the SMC receives all the crosstalk gains, iteratively calculate the PSDs, and imposes them to the user modems. If SCALE is used as originally proposed, with the protocol described in [9], where the SMC and the user modems exchange messages in a distributed way, the algorithm can be impractical. Considering that each DSL modem takes in average 30 seconds to update its own PSD mask (the retraining time), it would be necessary up to 20 minutes for the network to converge, with service interruptions in this period.

The 2SB algorithm has rather low complexity and satisfactory capacity performance, but it presents oscillations around the stationary point. At last, the ISB algorithm presented the best convergence performance among all DSM Lv. 2 algorithms considered in this work. Besides, it is well-known that ISB has the best rate performance, but it has much higher computational cost than the other discussed algorithms.

\section{FINAL REMARKS}

This work aimed at investigating the convergence of DSM algorithms. Essentially all DSM algorithms are iterative, and the specialized literature was missing a comparison with respect to convergence. This comparison must be done through simulations because there are no proofs of convergence for DSM algorithms.

The simulations conducted showed that DSM Lv. 1 algorithms have very fast convergence, and increasing the number of users results in a minimum influence over their convergence rate. This way, the best performance of those algorithms can be reached by 4 PSD mask adaptations per user at most.

For the spectrum balancing algorithms (DSM Lv. 2) considered in this work, a higher number of PSD mask adaptations are required (usually 20-40) in order to achieve the expected results in terms of capacity. This may restrict the algorithms to be used in centralized architectures, where all computations take place in the SMC.

Future works include the design of methods to improve the convergence of DSM algorithms Lv. 2, in order to reduce the 
user experience disturbance during DSM optimizations for the already deployed DSL equipments.

\section{REFERENCES}

[1] ANATEL, "Relatório anual 2008."

[2] J. Cook, R. Kirkby, M. Booth, K. Foster, D. Clarke, and G. Young, "The noise and crosstalk environment for ADSL and VDSL systems," IEEE Сотти. Mag., vol. 37, no. 5, pp. 73-78, May 1999.

[3] K. B. Song, S. T. Chung, G. Ginis, and J. M. Cioffi, "Dynamic spectrum management for next-generation DSL systems," IEEE Сотти. Mag., vol. 40, no. 10, pp. 101-109, Oct. 2002.

[4] J. Verlinden, T. Bostoen, and G. Ysebaert, "Dynamic spectrum management for digital subscriber lines - edition 2," June 2005.

[5] Dynamic Spectrum Management Technical Report, "ATIS committee NIPP pre-published document ATIS-PP-0600007," May 2007.

[6] W. Yu, G. Ginis, and J. M. Cioffi, "Distributed multiuser power control for digital subscriber lines," IEEE J. Select. Areas Commun., vol. 20, no. 5, pp. 1105-15, June 2002.

[7] R. Cendrillon and M. Moonen, "Iterative spectrum balancing for digital subscriber lines," in Communications, 2005. ICC '05. IEEE International Conference on, vol. 3, May 2005, pp. 1937-1941.

[8] R. Cendrillon, W. Yu, M. Moonen, J. Verlinden, and T. Bostoen, "Optimal multiuser spectrum balancing for digital subscriber lines," IEEE Transactions on Communications, vol. 54, no. 5, pp. 922-933, May 2006.

[9] J. Papandriopoulos and J. S. Evans, "Low-complexity distributed algorithms for spectrum balancing in multi-user DSL networks," in Communications, 2006. ICC '06. IEEE International Conference on, vol. 7, June 2006, pp. 3270 - 3275

[10] R. Moraes, A. Klautau, B. Dortschy, and J. Rius, "Semi-Blind Power Allocation for Digital Subscriber Lines," in Communications, 2008. ICC '08. IEEE International Conference on, 2008, pp. 1420-1425.

[11] M. N. Fonseca, D. M. Neves, A. C. Gomes, M. M. Conte, B. Dortschy, K. Ericson, J. R. I. Riu, E. Pelaes, and A. Klautau, "Evaluation of DSM performance with mixed DSL services and measured crosstalk channels," in 25th Simpósio Brasileiro de Telecomunicações (SBrT'07), Recife, Brazil, September. 2007.

[12] T. Starr, J. M. Cioffi, and P. J. Silverman, Understanding Digital Subscriber Line Technology. Prentice-Hall, 1999.

[13] T. Starr, M. Sorbara, J. M. Cioffi, and P. J. Silverman, DSL Advances. Prentice-Hall, 2003.

[14] R. Cendrillon, M. Moonen, J. Verliden, T. Bostoen, and W. Yu, "Optimal Multi-user Spectrum Management for Digital Subscriber Lines," in IEEE International Conference on Communications (ICC), vol. 1, 2004, pp. $1-5$.

[15] R. Cendrillon, M. Moonen, W. Yu, J. Verlinden, and T. Bostoen, "Proof of the optimality of optimal spectrum management," in ANSI T1E1.4 Working Group - DSL Access- Meeting, contrib. 2004-460, North Carolina, May 2004. 